\title{
ELEVADA FRECUENCIA DE DISLIPIDEMIA Y GLUCEMIA BASAL ALTERADA EN UNA POBLACIÓN PERUANA DE ALTURA*
}

\author{
Germán Málagaa ${ }^{1, a}$, Claudia Zevallos-Palacios²,b, María de los Ángeles Lazo ${ }^{2,3, b}$, Carlos Huayanay²,c

\begin{abstract}
RESUMEN
Se realizó un estudio transversal en la comunidad altoandina de Lari (3600 msnm) en Arequipa, Perú. Se evaluó el índice de masa corporal (IMC), niveles de glucosa y perfil de lípidos en 74 pobladores mayores de 18 años. La edad promedio fue de $51,7 \pm 18,0$ años, $62,2 \%$ fueron mujeres, el IMC promedio fue de $25,6 \pm 3,7$. Se encontró una prevalencia de hipercolesterolemia de $40,6 \%$, "HDL anormalmente bajo" en $77 \%$ de la población $(93,5 \%$ en mujeres frente a $50 \%$ en varones; $p<0,001$ ) y niveles elevados de LDL en el $71,7 \%$. La prevalencia de glucemia basal alterada fue del $27 \%$ y valores de glucosa $>126 \mathrm{mg} / \mathrm{dL}$ de $1,3 \%$. En conclusión, esta población altoandina tiene niveles elevados de glucemia basal alterada en ayunas, hipercolesterolemia y HDL anormalmente bajo. Se deben tomar en cuenta estos hallazgos para realizar intervenciones en poblaciones de altura para evitar futuras complicaciones cardiovasculares.
\end{abstract}

Palabras clave: Dislipidemias; Hipercolesterolemia; Prediabetes; Altitud, Perú (fuente: DeCS BIREME).

\section{HIGH FREQUENCY OF DYSLIPIDEMIA AND IMPAIRED FASTING GLYCEMIA IN A HIGH ALTITUDE PERUVIAN POPULATION}

\begin{abstract}
We performed a cross sectional study in Lari (3600 m), a highland rural community from Arequipa, Peru. We evaluated a body mass index (BMI), glycemia and lipid profile in 74 over 18 year persons. The mean age was $51.7 \pm 18.0$ years, $62.2 \%$ were women, mean of BMI was $25.6 \pm 3.7$. Prevalence of hypercholesterolemia was $40.6 \%$, "low HDL" in $77 \%$ of the population $(93.5 \%$ in women vs $50 \%$ in men, $p<0.001)$ and elevated level of LDL was $71.7 \%$. The prevalence of impaired fasting glycemia was $27 \%$. In conclusion, we found high prevalence of impaired fasting glycemia, hypercholesterolemia and especially "low HDL" in high altitude rural natives. These findings must be considered to realize interventions in high altitude populations to avoid future cardiovascular complications.
\end{abstract}

Key words: Dyslipidemias; Hypercholesterolemia; Prediabetes; Altitude; Peru (source: MeSH NLM).

\section{INTRODUCCIÓN}

Como consecuencia de los cambios demográficos y epidemiológicos de las últimas décadas, en el Perú se han producido importantes modificaciones en los perfiles de mortalidad y morbilidad, constituyendo las enfermedades cardiovasculares, el accidente cerebrovascular (ACV), las neoplasias malignas $y$ diabetes mellitus (DM) las principales causas de muerte y morbilidad en población adulta ${ }^{(1)}$.

Las enfermedades cardiovasculares (CV) se han convertido en una epidemia, lo que ha motivado que se constituyan en una de las áreas de investigación más extensa. El origen de estas enfermedades es multifactorial. El estudio INTERHEART demostró que los nueve factores de riesgo modificables para infarto de miocardio son los mismos para la mayoría de grupos poblacionales, estos son: tabaquismo, sedentarismo, consumo exagerado de alcohol, malos hábitos alimenticios, hipertensión, diabetes, obesidad abdominal, estrés psicosocial (laboral y familiar) y elevada razón de apolipoproteína B100/apolipoproteina A1 (2).

Existe la creencia de que el poblador de altura, dadas sus diferentes ocupaciones, dieta (predominio de carbohidratos) y actividad física, está protegido de las enfermedades $\mathrm{CV}$, lo que está respaldado por algunos estudios preliminares que reportaron niveles relativamente bajos de colesterol total (CT) y LDL y valores altos de colesterol HDL en pobladores de la altura, comparados con los que viven al nivel del mar ${ }^{(3)}$. En el estudio TORNASOL realizado en el $2006{ }^{(4)}$, se encontró, por medio de una encuesta, que las prevalencias de hipercolesterolemia y DM en mayores de 18 años eran similares entre las diferentes regiones, las ciudades estudiadas fueron: diez ciudades

\footnotetext{
Centro de Excelencia de Enfermedades Crónicas, Universidad Peruana Cayetano Heredia, Lima, Perú.

Facultad de Medicina, Universidad Peruana Cayetano Heredia, Lima, Perú.

Sociedad Científica de Estudiantes de Medicina Cayetano Heredia, Universidad Peruana Cayetano Heredia, Lima, Perú.

Médico internista, Magíster en medicina; ${ }^{b}$ Estudiante de Medicina; ${ }^{\circ}$ Estudiante de Tecnología Médica.

* El resumen fue presentado en el VIII Congreso Mundial de Medicina y Fisiología de Altura - Carlos Monge Cassinelli realizado del 8 al 12 de agosto del 2010 en Arequipa, Perú.
}

Recibido: 01-08-10 Aprobado: 27-10-10 
de la costa, seis ciudades por debajo de 3000 msnm y seis ciudades por encima de este nivel de la sierra, además de cuatro ciudades en la selva. La prevalencia de dislipidemia en la costa fue de $12,6 \%$ y en la sierra de $7,6 \%$; la prevalencia de diabetes fue de $3,3 \%$ siendo en la costa $4,3 \%$ y en la sierra $2,1 \%$; si a esto le aunamos los resultados de otro estudio realizado en $\mathrm{Cuzco}^{\left({ }^{5}\right)}$ en 1995 donde se encontró una prevalencia de ACV en mayores de 15 años (en 6,47 por 1000 habitantes) similar a la de países desarrollados, asociada con el sobrepeso y estilo de vida sedentario. Estos estudios nos sugieren que no podemos seguir afirmando que tenemos en la población andina bajas prevalencias de factores de riesgo o de enfermedad cardiovascular, pues constituyen un problema de salud pública en estas poblaciones.

Esta investigación se realizó en el marco del "XI Proyecto de Intervención Rural de Investigación y Saneamiento (IRIS)", actividad de extensión universitaria que realizan los estudiantes de ciencias de la salud, que combina acciones educativas, campañas de salud y proyectos de investigación en comunidades rurales ${ }^{(6)}$. El estudio buscó caracterizar los niveles de glucemia y perfil lipídico como factores de riesgo cardiovascular en la población mayor de 18 años del pueblo de Lari, provincia de Caylloma, Arequipa.

\section{EL ESTUDIO}

Se realizó un estudio transversal en el mes de agosto de 2009, en la comunidad de Lari, provincia de Caylloma, departamento de Arequipa, distrito ubicado a 3600 msnm, con 1379 habitantes para el año 2000 y una población mayor de 18 años de 600 pobladores. Los pobladores son aymaras y cuentan con una economía de subsistencia basada en la agricultura y ganadería. Sin embargo, el auge sostenido en el turismo de la zona, hasta hace pocos años casi sin contacto con el mundo occidental, así como el desarrollo hotelero y minero en zonas vecinas, ha provocado un cambio en sus costumbres, patrones alimentarios y actividad física.

Teniendo en cuenta la información del estudio PREVENCION ${ }^{(7)}$, con una prevalencia esperada de $5 \%$ para diabetes, con una confianza del $90 \%$ y un poder del $50 \%$, para una diferencia de $4 \%$ en la prevalencia de diabetes, era necesario recabar información de 73 pobladores. Los participantes fueron seleccionados al azar de una relación de pobladores mayores de edad obtenida por medio de un censo facilitado por las autoridades correspondientes, esta selección se hizo por medio de una tabla de números aleatorios. Cuando uno de los seleccionados no aceptaba participar o no cumplía con alguno de los criterios de inclusión, se elegía a la siguiente persona de la lista, hasta que acepte participar en el estudio. La tasa de aceptación fue de $90 \%$.

Se incluyó a pobladores mayores de 18 años de ambos sexos, con capacidad para leer y entender el consentimiento y que hayan aceptado participar voluntariamente. Se excluyó a las personas con historia o evidencia clínica de ascitis, hipotiroidismo, cirrosis, enfermedad hepática, insuficiencia renal crónica, embarazadas o con hijos menores de un año, o que se encuentren tomando progestágenos, esteroides anabólicos y corticoides.

A cada persona que aceptó participar se le tomó una muestra de $10 \mathrm{~mL}$ de sangre venosa en ayunas de al menos ocho horas entre las 07.00 y 09.00 horas. Se midió los niveles de glucosa, colesterol total, colesterol HDL, colesterol LDL y triglicéridos, con el método enzimático colorimétrico usando el equipo analizador semiautomático fotocolorímetro Microlab 200 (Merck). Adicionalmente, se pesó y talló a cada sujeto con un tallímetro y balanza calibrados, con los cuales se calculó el índice de masa corporal (IMC).

Se consideró colesterol elevado a valores $>200 \mathrm{mg} / \mathrm{dL}{ }^{(8)}$. Con relación al colesterol-LDL se asumió como nivel de riesgo $>100 \mathrm{mg} / \mathrm{dL}$. Para triglicéridos se consideró límite alto $\geq 150 \mathrm{mg} / \mathrm{dL}$. El colesterol-HDL se consideró alterado si era $<50 \mathrm{mg} / \mathrm{dL}$ en mujeres y $<40 \mathrm{mg} / \mathrm{dL}$ en varones, también se hizo el análisis considerando HDL $<40 \mathrm{mg} / \mathrm{dL}$ para ambos sexos ${ }^{(8)}$.

Se consideró glucemia basal alterada a valores $\geq 100 \mathrm{mg} / \mathrm{dL}$. Aquellos con valor $\geq 126 \mathrm{mg} / \mathrm{dL}$ fueron considerados con sospecha de diabetes ${ }^{(9)}$. Las personas que tomaban hipoglicemiantes fueron consideradas diabéticas.

Se consideró como sobrepeso a personas con valores de IMC > 25 y como obesidad si tenían más de 30 de IMC.

El estudio fue aprobado por el Comité de Ética de la Universidad Peruana Cayetano Heredia, y los resultados de las pruebas de laboratorio fueron entregados a los pacientes y derivados a los centros de salud para el manejo correspondiente.

Los datos fueron procesados con el programa STATA versión 10. Se determinó la prevalencia de cada factor de riesgo de acuerdo con las definiciones antes especificadas. Se calculó la media y la desviación estándar para variables numéricas, y porcentajes e intervalos de confianza para variables categóricas. Se usó la prueba de Chi cuadrado para comparar proporciones. Se consideró un $\mathrm{p}<0,05$ como significativo. 
Tabla 1. Características de los participantes incluidos en el estudio $(n=74)$.

\begin{tabular}{lcc}
\hline & $\mathbf{n}(\%)$ & IC $\mathbf{9 0 \%}$ \\
\hline Mujeres & $46(62,2)$ & $52,0-71,6$ \\
\hline Edad & $51,7 \pm 18,0$ & $48,2-55,1$ \\
\hline IMC * & $25,6 \pm 3,7$ & $24,9-26,3$ \\
Colesterol total >200mg/dL & $30(40,6)$ & $30,9-50,8$ \\
LDL > 100mg/dL & $53(71,6)$ & $61,8-80,1$ \\
\hline HDL <40mg/dL & $36(48,6)$ & $38,6-58,9$ \\
Triglicéridos > 150 mg/dL & $36(48,6)$ & $38,6-58,9$ \\
Glicemia en ayunas $>100 \mathrm{mg} / \mathrm{dL}$ & $22(29,7)$ & $21,1-39,7$ \\
\hline
\end{tabular}

* IMC: índice de masa corporal, se expresan en media \pm desviación estándar.

\section{HALLAZGOS}

Se incluyó 74 personas, 62,2\% eran mujeres (Tabla 1), el promedio de edad fue de 56,0 en mujeres y de 49,23 en varones. Los valores promedios de IMC fueron de 26,07 y 24,8 en mujeres y varones respectivamente ( $p>0,05)$. La prevalencia de sobrepeso fue de $41,3 \%$, mientras que la de obesidad fue de $8,7 \%$.

Se halló valores de colesterol mayores de $200 \mathrm{mg} / \mathrm{dL}$ en el 40,6\%. En el 9,3\% de pobladores el valor de colesterol fue mayor de $240 \mathrm{mg} / \mathrm{dL}$, no se encontró diferencias según sexo (Tabla 2 ). En el $77 \%$ de la población se encontró niveles bajos de HDL, las mujeres tuvieron una prevalencia de HDL bajo menor que los varones $(p<0,001)$. Usando la definición de $\mathrm{HDL}<40 \mathrm{mg} / \mathrm{dL}$, encontramos un $48,6 \%$ con esta condición.

En $71,7 \%$ de los pobladores se halló niveles de colesterol LDL mayores a $100 \mathrm{mg} / \mathrm{dL}, 35,2 \%$ mayores a $130 \mathrm{mg} /$ dL y en $14,9 \%$ por encima de $160 \mathrm{mg} / \mathrm{dL}$, sin diferencia entre sexos $(71,4 \%$ en varones y $71,7 \%$ en mujeres).

En 48,6\% de individuos se presentó niveles de triglicéridos mayor de $150 \mathrm{mg} / \mathrm{dL}$, la mitad de ellos muestra niveles entre 150 a 199 mg/dL y el resto entre 200 y 399 mg/dL.

Tabla 2. Prevalencias de valores alterados de glicemia y perfil lipídico entre hombres y mujeres*.

\begin{tabular}{lcc}
\hline & $\begin{array}{c}\text { Mujeres } \\
\mathbf{n = 4 6}\end{array}$ & $\begin{array}{c}\text { Hombres } \\
\mathbf{n = 2 8}\end{array}$ \\
\hline Colesterol total $>200 \mathrm{mg} / \mathrm{dL}$ & $18(38,3)$ & $13(46,3)$ \\
$\mathrm{LDL}>100 \mathrm{mg} / \mathrm{dL}$ & $33(71,7)$ & $20(71,4)$ \\
$\mathrm{HDL}$ bajo†‡ & $43(93,5)$ & $14(50,0)$ \\
$\mathrm{HDL}<40 \mathrm{mg} / \mathrm{dL}$ & $21(47,8)$ & $14(50,0)$ \\
Triglicéridos $>150 \mathrm{mg} / \mathrm{dL}$ & $22(47,8)$ & $14(50,0)$ \\
Glucemia en ayunas $>100 \mathrm{mg} / \mathrm{dL}$ & $11(24,0)$ & $9(32,1)$ \\
\hline
\end{tabular}

* Los valores representan frecuencia (porcentajes)

† Se consideró $<50 \mathrm{mg} / \mathrm{dL}$ en varones y $<40 \mathrm{mg} / \mathrm{dL}$ en mujeres. $\ddagger \mathrm{p}<0,001$.
El $27,0 \%$ de los pobladores presentó glucemia basal alterada y el $1,3 \%$ presentó niveles de sospecha de diabetes, sin diferencias significativas según sexo $(p>0,05)$.

\section{DISCUSIÓN}

Se encontró una elevada proporción de pobladores con hallazgo de "HDL bajo" (hasta el 77\%), especialmente en las mujeres (93,5\%). Estos valores exceden a los hallados en diferentes estudios realizados en pobladores de altura y al nivel del mar. Estudios iniciales reportaron niveles relativamente bajos de colesterol total (CT) y LDL y valores altos de colesterol HDL en pobladores de altura, comparados con los que viven al nivel del mar (3). En 1993, Bellido et al. ${ }^{(10)}$ reportaron en Viacha (3876 msnm) valores de CT, LDL y triglicéridos bajos, pero una alta prevalencia de "HDL bajo". Posteriormente, Mohanna et al. (11), en San Pedro de Cajas (4100msnm) en el año 2006, encontraron resultados similares a los nuestros, es decir, prevalencias más altas de "HDL bajo" en mujeres $(45,3 \%$; $p<0,001)$.

Estos resultados son concordantes con los del estudio PREVENCION realizado en la ciudad de Arequipa a 2300 msnm, en el que se reportó una prevalencia de "HDL bajo" en $60,9 \%$ de mujeres ${ }^{(7)}$. Nuestro estudio también halló esta diferencia entre sexos, todo esto sugiere que la prevalencia tan alta de HDL bajo y predominante en mujeres, es consistente en población de altura.

Este hallazgo de "HDL bajo", ya había sido reportado en algunos estudios en la costa y en poblaciones latinas, pero no se le ha puesto el énfasis suficiente, Seclén et al. en un estudio hecho en Lima, en pacientes con síndrome metabólico, reportó "HDL bajo" en 55,0\% de hombres y $75,4 \%$ de mujeres ${ }^{(12)}$.

En el estudio CARMELA realizado en siete ciudades latinoamericanas ${ }^{(13)}$, el reporte de "HDL bajo" ( $<40 \mathrm{mg} / \mathrm{dL}$ ) fue mayor en Lima $(56,9 \%)$ que en otras ciudades como Barquisimeto (52,2\%), Bogotá (45,6\%), Ciudad de México $(22,6)$, Quito $(21,6 \%)$, Santiago de Chile $(21,2 \%)$ y en Buenos Aires $(16,9)$ y menores a las encontradas por nosotros.

Estos resultados son de especial importancia si tenemos en cuenta lo demostrado en diversos estudios poblacionales donde se concluye de manera consistente que el nivel de HDL es un factor predictor independiente inverso de enfermedad cardiovascular ${ }^{(14)}$. En el estudio Framingham, el nivel HDL fue más potente como factor de riesgo para enfermedad coronaria que el nivel de LDL (15). 
De acuerdo con la información presentada, consideramos que en poblaciones de altura o algunas latinas, destacaría la alta prevalencia de "HDL bajo", lo que debe ser investigado en estudios realizados en altura y con tamaños muestrales más grandes que pongan énfasis en este aspecto, además de considerar la dieta de la población.

En nuestro estudio, además, tuvimos niveles más altos de colesterol total $(40,6 \%)$ y LDL $(71,7 \%>100 \mathrm{mg} / \mathrm{dL}$ y $35,2 \%>130 \mathrm{mg} / \mathrm{dL}$ ) comparados con estudios previos tanto en altura como a nivel del mar (11-13). Mohanna reportó hipercolesterolemia en $34,3 \%$ y LDL elevado (> 130mg/dL) en $11,8 \%{ }^{(11)}$. En el estudio CARMELA se encontró $14,2 \%$ de hipercolesterolemia en varones y $13,6 \%$ en mujeres, las prevalencias de CT y LDL elevados fueron de 5,7 y $9,8 \%$ en Barquisimeto; 11,6 y $17,7 \%$ en Lima; 11,7 y $19,1 \%$ en Bogotá; 15,3 y $19,9 \%$ en Santiago de Chile; 16,4 y 25,6\% en México DF; 18,7 y $24,7 \%$ en Buenos Aires y 20,2 y $23,9 \%$ en Quito ${ }^{(13)}$.

Una explicación a la alta prevalencia de hipertrigliceridemia y colesterol HDL bajo, podría deberse al gran consumo de carbohidratos que caracteriza a la población andina, ya que una dieta rica en azucares está relacionada con hipertrigliceridemia y dietas bajas en grasas y altas en carbohidratos conllevan a bajos niveles de HDL ${ }^{(16)}$.

En relación con la glucemia basal alterada (glucosa $>100$ $\mathrm{mg} / \mathrm{dL}$ ), hallamos una prevalencia de $27 \%$, mientras que en el estudio PREVENCION la prevalencia fue alrededor de $5 \%{ }^{(7)}$. En otras latitudes, se describen valores de $29 \%$ en nativos canadienses, $15,3 \%$ en haitianos y $28 \%$ en mexicanos ${ }^{(17)}$. Sin embargo, la prevalencia de diabetes hallada es baja (1,3\%), más bajo de lo hallado en general en otros estudios y discordante con la prevalencia de glucemia basal alterada. No tenemos una clara explicación ni referencias que respalden este hallazgo.

El estudio tiene limitaciones, debido a que la muestra no permite establecer estratificación por grupos etarios ni por otras variables, asimismo, el poder de nuestro estudio no fue el adecuado para encontrar diferencias estadísticamente significativas menores de $4 \%$. No se midió la actividad física, el tipo de alimentación y otras variables que estén relacionadas con estos factores de riesgo cardiovascular.

No tenemos una clara explicación para nuestros hallazgos, pues podrían ser aspectos dietéticos, ambientales, genéticos (étnicamente, la población de Lari, proviene de los Collaguas de procedencia Aymara) o será que los valores que aceptamos como "normales" en lo que a perfil lipídico se refiere, no son aplicables a pobladores de altura.
Estos resultados nos permiten afirmar que no podemos seguir en la creencia de que en los pobladores de altura no son frecuentes los factores de riesgo cardiovascular, lo que debe llevar a reevaluar las prioridades de atención a corto y largo plazo en estos grupos poblacionales en relación con estas enfermedades. Por lo que se debe poner especial énfasis en educación y en medias de prevención, antes de enfrentar serios problemas cardiovasculares en una población económicamente muy deprimida.

\section{Financiamiento}

Facultad de Medicina y Escuela de Tecnología Médica de la Universidad Peruana Cayetano Heredia.

\section{Conflictos de Interés}

Los autores declaran no tener conflictos de interés en la publicación de este artículo.

\section{REFERENCIAS BIBLIOGRÁFICAS}

1. Seclén S. Enfermedades crónicas no transmisibles en la población peruana. Rev Med Hered. 1995; 6(4): 161-162.

2. Yusuf S, Hawken S, Ounpuu S, Dans T, Avezum A, Lanas $\mathbf{F}$, et al. Effect of potentially modifiable risk factors associated with myocardial infarction in 52 countries (the INTERHEART study): case-control study. Lancet. 2004;364(9438):937-52.

3. Piedra A, Marticorena E, Muro M, Díaz C, Picón-Reategui E, Liñan A, et al. Lípidos en individuos normales de altura y de nivel del mar a propósito del proceso de envejecimiento en la altura. Arch Biol Andina. 1981;11:20-25.

4. Segura L, Regulo C, Parodi J. Factores de riesgo de las enfermedades cardiovasculares en el Perú (Estudio TORNASOL). Rev Per Cardiol. 2006;32(2):82-128.

5. Jaillard AS, Hommel M, Mazetti P. Prevalence of stroke at high altitude $(3380 \mathrm{~m})$ in Cuzco, a town of Peru. A population-based study. Stroke. 1995;26(4):562-68.

6. Rivas-Nieto AC, Curioso WH, Guillén C. Participación estudiantil en proyectos de intervención rural en salud: la experiencia IRIS-X en Perú. Rev Peru Med Exp Salud Publica. 2009;26(3):387-94.

7. Medina-Lezama J, Zea-Díaz H, Morey-Vargas OL, Bolaños-Salazar JF, Muñoz-Atahualpa E, PostigoMacDowall M, et al. Prevalence of the metabolic syndrome in Peruvian Andean Hispanics: The PREVENCION study. Diabetes Res Clin Pract. 2007;78(2):270-81.

8. National Cholesterol Education Program (NCEP) Expert Panel on Detection, Evaluation, and Treatment of High Blood Cholesterol in Adults (Adult Treatment Panel III).Third Report of the National Cholesterol Education Program (NCEP) Expert Panel on Detection, Evaluation, and Treatment of High Blood Cholesterol in Adults (Adult Treatment Panel III) Final Report. Circulation. 2002;106:3143-421.

9. Petersen JL, McGuire DK. Impaired glucose tolerance and impaired fasting glucose--a review of diagnosis, 
clinical implications and management. Diab Vasc Dis Res. 2005;2:9-15

10. Bellido D, Barragán M, Aguilar M, Colque N. Perfil lipídico de la población de Viacha. Acta Andina.1993;2(1):28-29.

11. Mohana S, Baracco R, Seclén S. Lipid profile, waist circumference, and body mass index in a high altitude population. High Alt Med Biol. 2006;7(3):245-55.

12. Seclén S, Villena A, Larrad MT, Gamarra D, Herrera B, Pérez CF, et al. Prevalence of the metabolic syndrome in the mestizo population of Peru. Metab Syndr Relat Disord. 2006;4(1):1-6.

13. Vinueza R, Boissonnet CP, Acevedo M, Uriza F, Benitez FJ, Silva $\mathbf{H}$, et al. Dyslipidemia in seven Latin American cities: CARMELA study. Prev Med. 2010;50(3):106-11.

14. Assmann G, Schulte H, von Eckardstein A, Huang Y. High-density lipoprotein cholesterol as a predictor of coronary heart disease risk: the PROCAM experience and pathophysiological implications for reverse cholesterol transport. Atherosclerosis 1996;124:S11-S20.
15. Gordon DJ, Probstfield JL, Garrison RJ, Neaton JD, Castelli WP, Knoke JD, et al. High-density lipoprotein cholesterol and cardiovascular disease: four prospective American studies. Circulation. 1989;79(1):8-15.

16. Bierman E. Carbohydrate and sucrose intake in the causation of atherosclerotic heart disease, diabetes mellitus, and dental caries. Am J Clin Nutr. 1979;32(12):2644-47.

17. Delisle H, Désilets MC, Vargas ER, Garrel D. Metabolic syndrome in three ethnic groups using current definitions. Appl Physiol Nutr Metab. 2008;33(2):356-60.

Correspondencia: Germán Málaga.

Dirección: Calle Chavín 159. CC Monterrico. Lima 33.

Teléfono: (51) 992-768-300

Correo electrónico: gmalaga01@gmail.com

\section{www.scielosp.org}

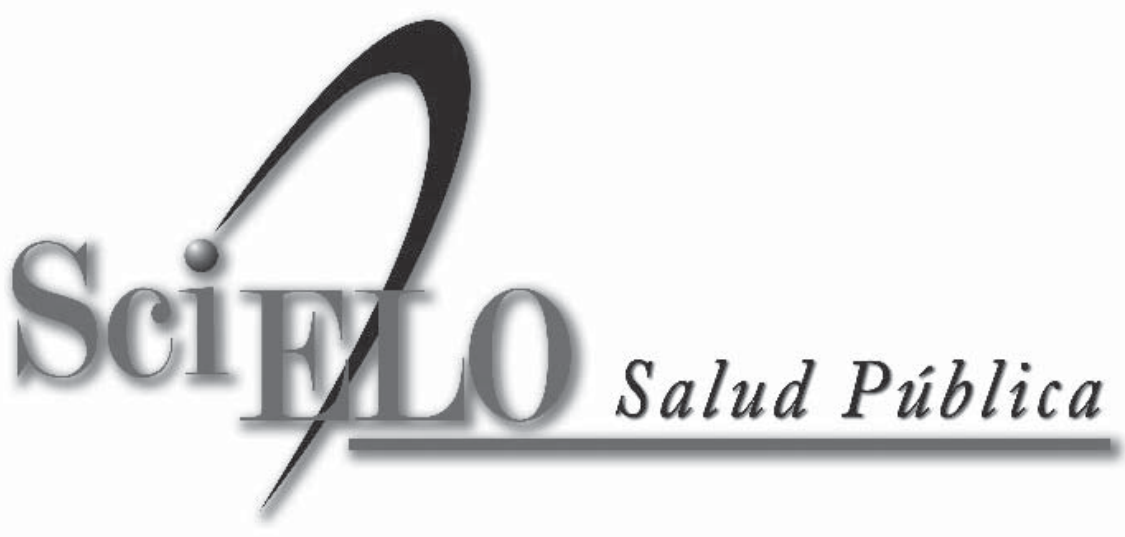

